\title{
Metastable fcc Structure of Ti-Mg Alloy Synthesized by Mechanical Alloying
}

Armando Tejeda-Ochoa ${ }^{1}$, Caleb Carreño-Gallardo ${ }^{1}$, Nagamasa Kametani ${ }^{2}$, Shota Yotsumoto ${ }^{2}$, Nozomu Adachi $^{2}$, Yoshikazu Todaka ${ }^{2}$ and Jose Martin Herrera-Ramirez ${ }^{1}$

${ }^{1}$ Centro de Investigacion en Materiales Avanzados S.C., Chihuahua, Chihuahua, Mexico, ${ }^{2}$ Toyohashi University of Technology, Toyohashi, Aichi, Japan

Ti-Mg alloys have great potential for different industries where good strength-to-weight ratios are desired. In the reported binary phase diagram [1], the mutual solubility between $\mathrm{Ti}$ and $\mathrm{Mg}$ is less than 2 at\%. Furthermore, the difference in their melting points makes them impossible to produce by conventional metallurgy. It has been demonstrated that mechanical alloying (MA) is an excellent technique for extending terminal solid solubility. Furthermore, MA can be used to induce chemical reactions at well below temperatures than normally required [2]. Therefore, equilibrium and non-equilibrium alloy powders with extremely fine microstructures can be obtained by MA.

In this work, the Ti50Mg50 system was investigated. For this purpose, elemental powders Ti $99.5 \%$ and Mg $99.8 \%$ both with a particle size of $\sim 45 \mu \mathrm{m}$ provided by Alfa Aesar were weighted inside a globe box filled with argon gas. A Spex 8000M high-energy ball mill (SBM) with hardened steel vial and balls was used to synthesize the alloy during $25 \mathrm{~h}$. The ball-to-powder ratio was settled at 10:1. Stearic acid (4 wt\%) was used as a process control agent to avoid the cold welding during the process. The milling was performed under argon atmosphere. The alloyed powders were cold consolidated and then characterized by X-ray diffraction (XRD) using a Rigaku Ultima IV diffractometer with $\mathrm{Cu}-\mathrm{K} \alpha(0.1542 \mathrm{~nm})$. A high resolution transmission electron microscope (JEOL JEM2200FS+CS) with an accelerating voltage of 200 $\mathrm{kV}$ was used to confirm the phase transformation and measure lattice parameters.

Fig. 1 displays XRD patterns of the raw material and the Ti50Mg50 system processed by SBM. The unprocessed $\mathrm{Ti}$ and $\mathrm{Mg}$ possess an hcp structure with $\mathrm{a}=0.295 \mathrm{~nm}$ and $\mathrm{c}=0.468 \mathrm{~nm}$ for Ti, and $\mathrm{a}=0.321$ $\mathrm{nm}$ and $\mathrm{c}=0.521 \mathrm{~nm}$ for $\mathrm{Mg}$. After $25 \mathrm{~h}$ of SBM, wide peaks can be observed in the XRD pattern of the Ti50Mg50 alloy. The most intense peaks correspond to the new metastable fcc phase, with the presence of remaining peaks of $\mathrm{Mg}$ hcp. The lattice parameter of the alloy was calculated by the SAED patterns, due to the uncertain position of the fcc peaks in the XRD diffractogram (Fig. 1).

The heavy plastic deformation during the ball milling results in an increase of punctual defects. The phase transformation hep to fcc is linked to stacking faults produced during the milling processing. This metastable fcc phase could be stabilized when the grain size is below $20 \mathrm{~nm}$, according to Zheng et al. and Xiong et al. [3, 4].

Transmission electron microscopy results are presented in Fig. 2. The bright field image (Fig. 2a) shows the presence of nanograins (grain size below $20 \mathrm{~nm}$ ) well distributed along the sample. The SAED pattern (Fig. 2b) reveals characteristic rings of a polycrystalline material and shows a single phase. The pattern indexing confirms the formation of the metastable fcc phase with $\mathrm{a}=0.439 \mathrm{~nm}$. The high resolution image in Fig. 2c shows a nanograin $(\sim 6 \mathrm{~nm})$ corresponding to the fcc phase.

The composition Ti50Mg50 was successfully synthesized by mechanical alloying. As a result, the solid solubility limit was increased and a nanostructured material with a new metastable fcc phase was obtained. The fcc phase was stabilized by the introduction of stacking faults at regular intervals when the grain size is kept below $20 \mathrm{~nm}$. 


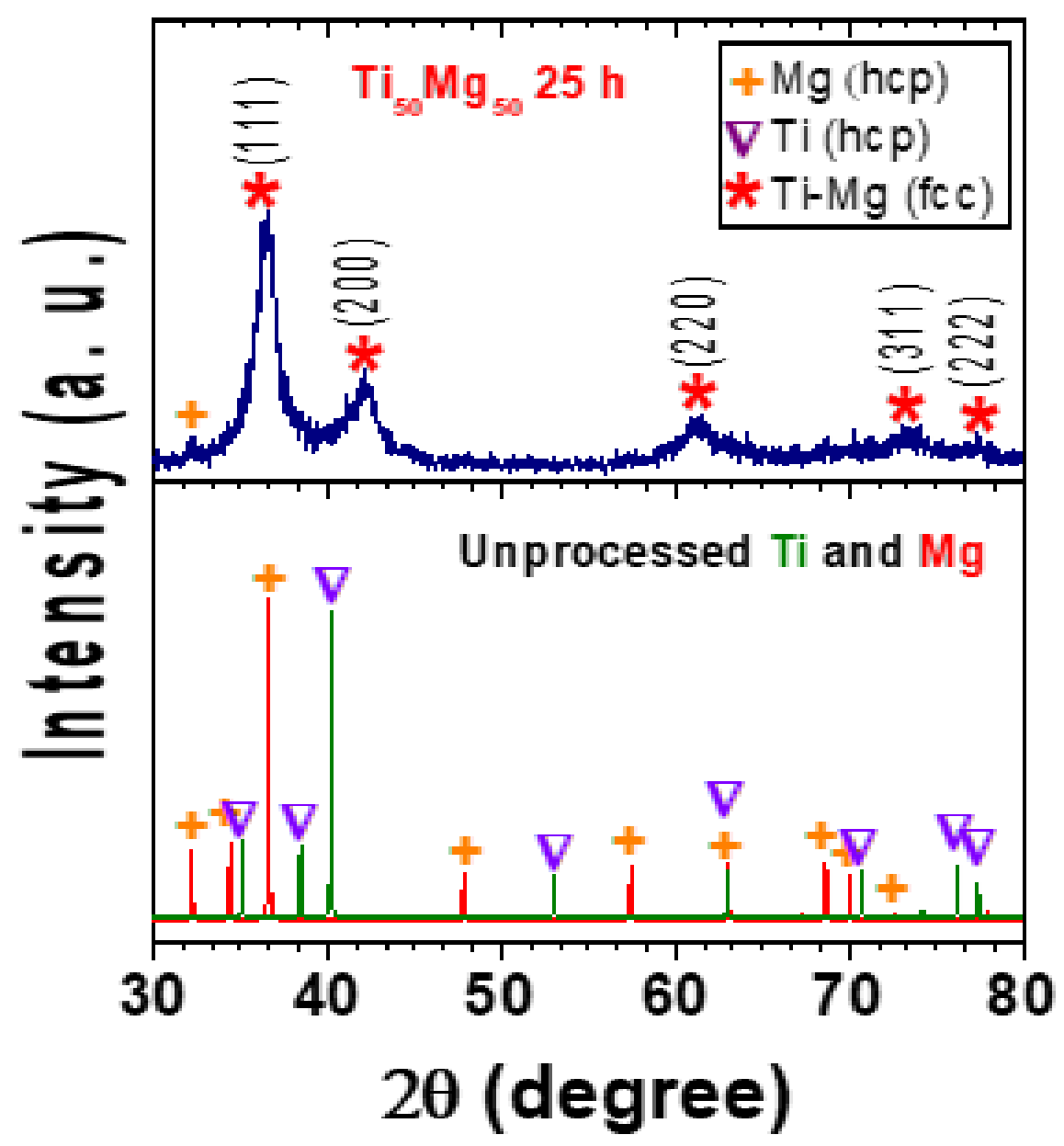

Figure 1. XRD patterns of the Ti50Mg 50 alloy before and after processing by SBM.
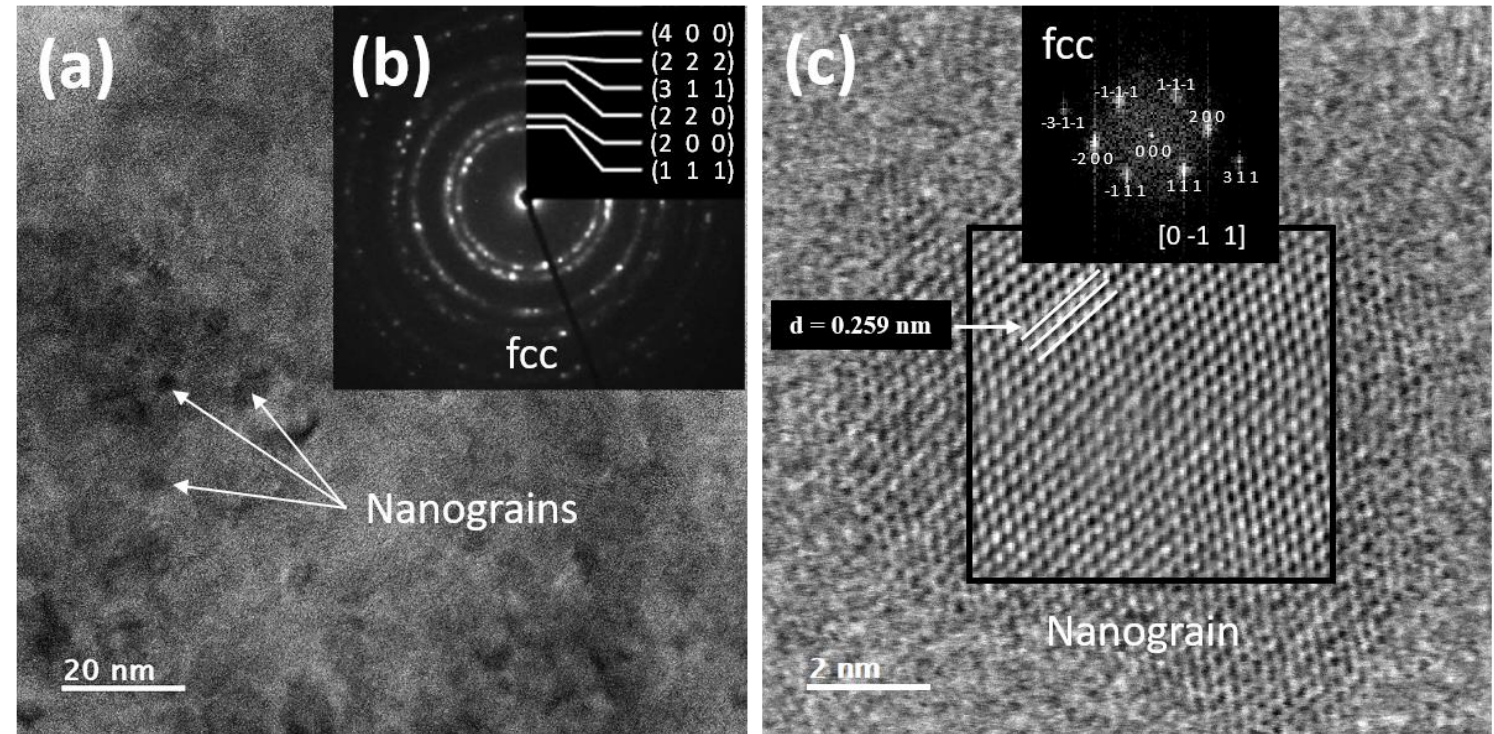

Figure 2. TEM analysis for the Ti50Mg 50 alloy processed by SBM for $25 \mathrm{~h}$ : a) bright field image b) indexed SAED pattern, and c) high resolution image. 


\section{References}

1. A Nayeb-Hashemi. Phase diagrams of binary magnesium alloys. ASM International, Metals Park, Ohio 44073, USA, 1988. 370, 1988.

2. Cury Suryanarayana. Mechanical alloying and milling. Progress in materials science, 46(1-2):1-184, 2001.

3. Zheng, G.P., Y.M. Wang, and M. Li, Atomistic simulation studies on deformation mechanism of nanocrystalline cobalt. Acta Materialia, 2005. 53(14): p. 3893-3901.

4. Xiong, S., et al., Size and shape dependent Gibbs free energy and phase stability of titanium and zirconium nanoparticles. Materials Chemistry and Physics, 2010. 120(2): p. 446-451. 